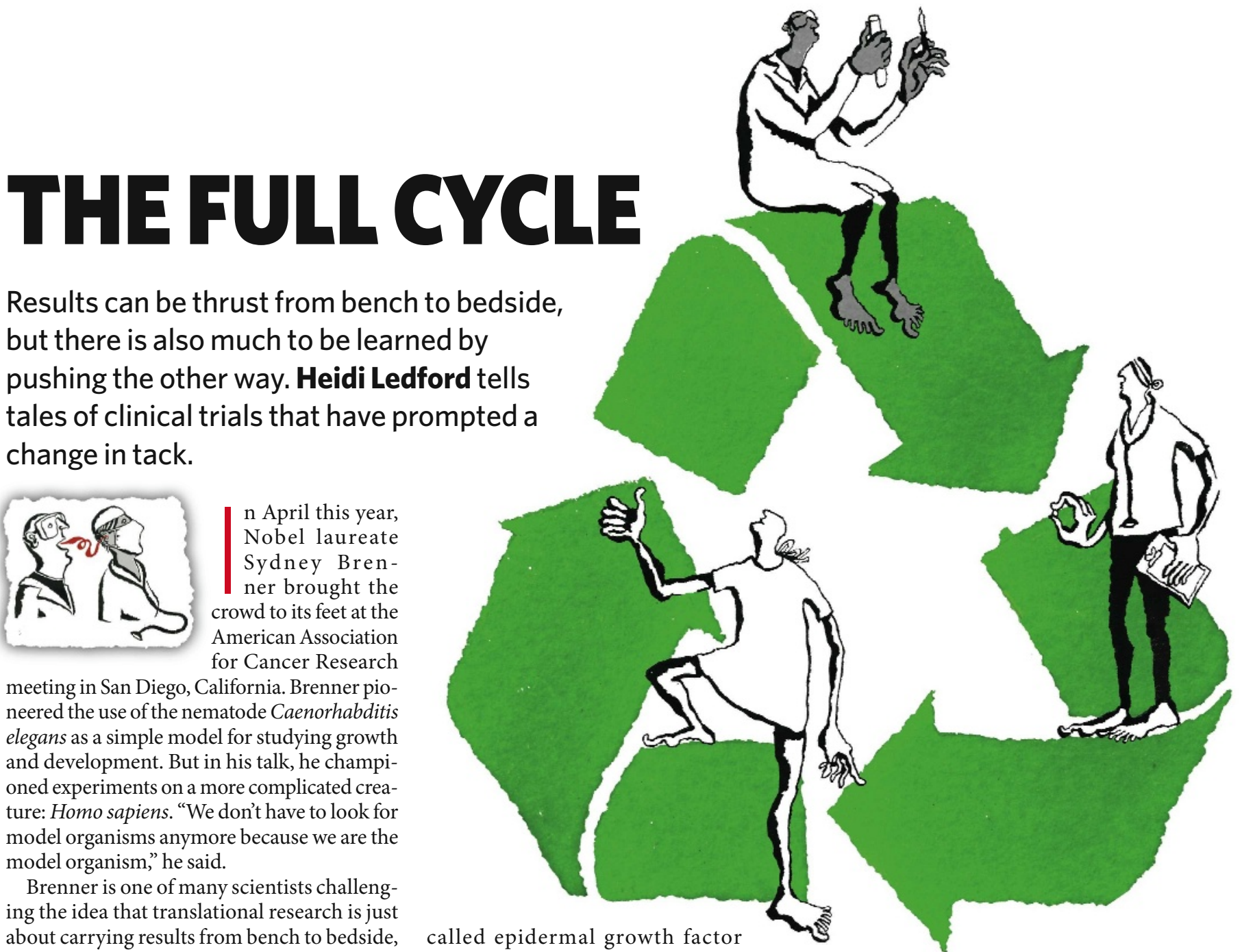

called epidermal growth factor receptor (EGFR).

Several types of tumour churn out higher than normal levels of EGFR, and gefitinib was designed to block the receptor and prevent further tumour growth. In 2003, the US Food and Drug Administration conditionally approved the drug, marketed by AstraZeneca as Iressa, to treat a severe lung cancer called non-smallexperiment in its next iteration.

Reverse translation of this type comes with its own challenges, such as gaining access to clinical samples. And only certain trials, for example those the research community has already invested in, tend to get this type of scientific scrutiny. Below, Nature recounts three stories in which results from human experiments inspired new avenues of research.

\section{A moving target}

t was a front-page article in The Boston Globe that first caught cancer researcher Daniel Haber's attention. The 2003 article $^{1}$ described the strange results from an experimental cancer drug called gefitinib, one of the first generation of 'smart drugs' designed to target a specific protein, in this case one
"We don't have

to look for model

organisms anymore

because we are the model organism."

- Sydney Brenner cell carcinoma. And in some cases - typically non-smokers, women and Asians - the drug yielded dramatic results, seemingly eradicating signs of the disease from patients whose illness was thought to be terminal. "The response was magical," says Haber, director of the Massachusetts

General Hospital Cancer Center in Boston.

But in other patients, as the Globe article explained, gefitinib was ineffective. Trials completed after the drug's approval showed that it failed to improve patients' survival when averaged across all individuals ${ }^{2}$. Regulators took the drug off the US market in 2005, although it is still approved in some other countries, and for certain exceptional cases in the United States.

To many, the drug's failure was a bitter disappointment. But Haber was intrigued and wondered whether there was a molecular explanation for the discrepancy $\stackrel{\circ}{9}$ in responses between one patient and the next. Down the road at the Dana Farber Cancer Research Institute, Matthew Meyerson and his colleagues were wondering the same thing. Neither group initially thought to look for answers in the sequence of the EGFR gene: the experiment was so obvious that both assumed it had already been done. "It was naivety," says Meyerson.

In 2004, Haber, Meyerson, and William Pao at the Memorial Sloan-Kettering Cancer Center in New York, independently published results showing that most tumours that responded to gefitinib harboured mutations in EGFR that rendered the protein more sensitive than usual to the drug ${ }^{3-5}$. As a result of these findings, researchers have developed genetic tests for EGFR mutations, and several clinical trials are now under way to determine whether the drug is effective when it is given only to the patients with a mutated receptor.

But there was another puzzle to solve. Early trials had shown that even those who did initially respond to the EGFR inhibitor soon become resistant, many within a year of starting treatment. By genetic analysis of tumour samples and cancer cell lines, researchers have found that these resistant tumours acquire secondary mutations that render the drug impotent. 
Sometimes, the culprit is a second mutation in EGFR that stops the drug from binding to the protein; at other times, a gene called MET is also amplified, which allows the tumour cells to multiply even when EGFR is not working ${ }^{6}$. Occasionally both EGFR mutations and MET amplification arise in different cells within the same patient, illustrating how cancer cells will use every genetic trick in the book to continue growing. Findings such as these partly inspired the Cancer Genome Project, which aims to sequence genes from multiple cancers to reveal their genetic idiosyncrasies. And early clinical trials are under way to test whether gefitinib-resistant tumours that have accumulated a secondary mutation can be tackled with alterna-

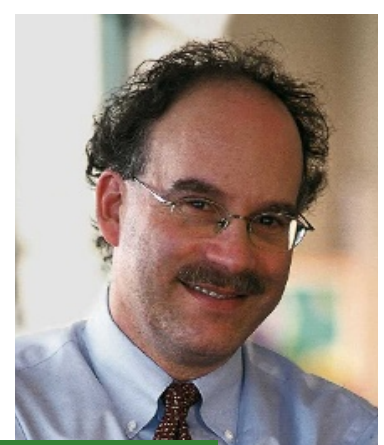

"The response to gefitinib was magical." - Daniel Haber a mutation in a gene, called IL2RG, that is required for the immune system to generate working $\mathrm{T}$ cells and other cells in the immune system. The condition is commonly called 'bubble boy disease' because babies with X-SCID must live in sterile environments to avoid lethal infections.

At first, the two X-SCID trials, in France and Britain, were heralded as the clearest success in the controversial gene-therapy field. Most patients developed a functional immune system, and the first recipients now live a normal life, says Marina Cavazzana-Calvo, a researcher at the Neckers Children's Hospital in Paris, France, who helped conduct the French trial. The technique relied on a retrovirus to shuttle a functional copy tive drugs, such as EGFR inhibitors that bind more tightly to the protein and are unaffected by the mutations.

Few failed drugs receive so much scrutiny. Gefitinib was a special case because it had been designed specifically for a target, and because it produced such a 'magical' response in some patients.

One of the major obstacles in bedside-tobench research is obtaining high-quality tissue samples from trials. Few physicians are willing to collect the multiple invasive biopsies that are needed to determine the molecular changes in a tumour as it evolves, but that offer no direct benefit to the patient. Meyerson had to track down the investigators who had tested the drug and apply for separate approval to use the samples from the many ethics committees who approved the gefitinib study. "I didn't understand how hard it was to get samples from a clinical trial at that time," he says.

\section{Tale of the unexpected}

/hen leukaemia first developed in a child given gene therapy, there was still hope that it was just a coincidence. "We didn't know what to make of it," says Brian Sorrentino, who directs the gene-therapy programme at St Jude Children's Research Hospital in Memphis, Tennessee. "Then the second case came, and it was clear this was going to be a recurring problem."

Since 2002,5 of the 21 children who received a high-profile, experimental gene-therapy treatment for a disease called X-linked severe combined immunodeficiency (X-SCID) have developed leukaemia. X-SCID is caused by of $I L 2 R G$ into the patient's bone marrow stem cells, from which immune cells are generated. Researchers expected that the retrovirus would integrate into the patient's genome at random. But shortly after the two trials started, Christopher Baum of Hannover Medical School in Germany, and his colleagues published a short report indicating that the virus preferentially inserted itself next to a cancer-causing gene, causing leukaemia in mouse models. "The initial reaction was that our mouse model would not be relevant to the clinics," Baum says.

Once the third case of leukaemia came to light in 2005, the French trial was put on hold, sending a chill through the entire gene-therapy community. "It was a difficult time," says immunologist Frank Staal, who studies gene therapy

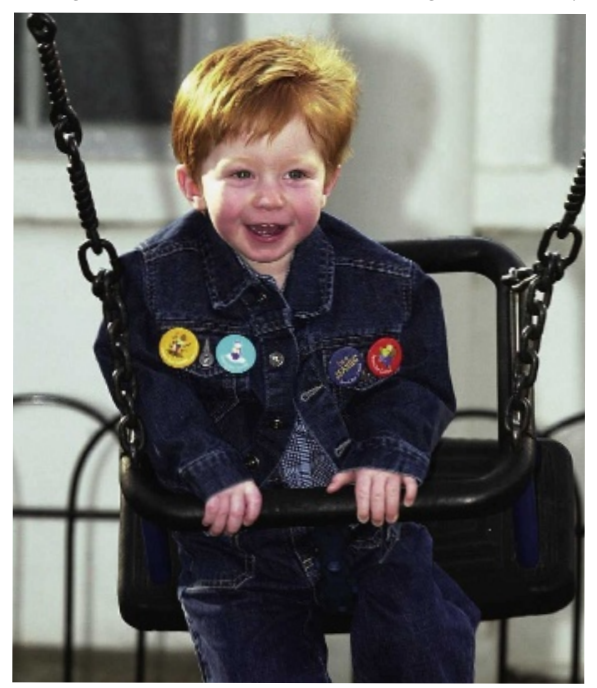

Gene therapy helped some children who were born without a working immune system. at the Erasmus University Medical Center in Rotterdam, the Netherlands. For many clinical trials, such a disaster would mark an immediate end to the research. But because this therapy had looked so promising and because the disease is so devastating, researchers were anxious to find out what had gone wrong.

Using a method to isolate and amplify only the regions of DNA that surrounded the virus, scientists have found that the insertions were far from random in the bone-marrow stem cells. The virus had multiplied and slotted into hundreds of different sites, preferring to settle near highly expressed genes ${ }^{8,9}$. In the French trial, some of the patients with leukaemia had viral inserts near possible cancer-causing genes such as LMO2, which is involved in blood-cell formation ${ }^{10}$. The researchers suspected that genetic elements in the virus that were used to activate IL2RG also stimulated expression of LMO2 and other genes nearby the virus' insertion site. This probably caused the proliferation of T cells that caused the leukaemia.

Since then, researchers have been trying to get around this by modifying the viruses used to transfer the gene. Adrian Thrasher at University College London has designed a vector with genetic control regions that are less likely to activate nearby genes and that contains a 'kill switch' to prevent it from replicating once it has inserted into the genome ${ }^{11}$. Pending final approval, this vector will be used in the next round of genetherapy trials for X-SCID, says Thrasher, who is one of those leading the trial.

Of the five children who developed leukaemia, four were successfully treated and their gene-therapy-repaired immune systems remained intact. Sorrentino notes that if the gene therapy itself had not been so successful, the community might not have rallied so readily to fix the leukaemia problem. "At the time it felt terribly depressing," he says. "But we've worked through that and now I feel very enthusiastic."

\section{Test shot}

$\mathrm{B}$ the time Merck's HIV $B$ vaccine candidate made it into a phase II trial, there was no shortage of strong opinions about its probable fate. Over the past few decades, the 
